\title{
EXPERIENCE IN TREATING LONG-TERM EFFECTS OF CONCUSSIONS AND PTSD USING MANUAL THERAPY COMBINED WITH SHOCK WAVE THERAPY
}

\author{
Sergii Frank \\ Ukraine, Kyiv, Medical Center "Ukrainian Trauma Center" \\ Orcid ID: https://orcid.org/0000-0002-0053-6898.
}

DOI: https://doi.org/10.31435/rsglobal_ws/30042019/6470

\section{ARTICLE INFO}

Received: 19 February 2019

Accepted: 11 April 2019

Published: 30 April 2019

\section{KEYWORDS}

long-term effects of concussions, mTBI,

PTSD,

manual therapy,

shock wave therapy

\begin{abstract}
The purpose of this article is the desire of the author, based on his previous multiple years' experience in treating the effects of mTBI, as well as his experience in treating the effects of combat concussions, to offer, in addition to the methods already described, his own vision of how to deal with the problem at hand. The method proposed lies in complex application of manual therapy combined with shock wave therapy. Their combined power, when applied within a unified method of treatment, enhances the effect of each of them, creating a synergy that allows to achieve qualitatively better and significantly more successful clinical results.
\end{abstract}

Citation: Sergii Frank. (2019) Experience in Treating Long-Term Effects of Concussions and Ptsd Using Manual Therapy Combined with Shock Wave Therapy. World Science. 4(44), Vol.2. doi: 10.31435/rsglobal_ws/30042019/6470

Copyright: (C) 2019 Sergii Frank. This is an open-access article distributed under the terms of the Creative Commons Attribution License (CC BY). The use, distribution or reproduction in other forums is permitted, provided the original author(s) or licensor are credited and that the original publication in this journal is cited, in accordance with accepted academic practice. No use, distribution or reproduction is permitted which does not comply with these terms.

Introduction. The military conflict that arose in 2014 and continues today in Ukraine has resulted in numerous human casualties, including thousands of wounded. According to UN reports, from April 2014 and until August 2017 at least 34,766 casualties resulted by hostilities were recorded in the Donbas region, of which 10,225 were killed and 24,541 wounded [19, p. 7]. This conflict involves the use of heavy weapons, most of which are explosive weapons with a wide radius of destruction, such as artillery and mortars, as well as multiple-launch rocket systems [19, p. 4]. The most common damage from such weapons includes mine-explosive fragmentation injuries with lesions of limbs, neck, head, less often those of the trunk, as well as concussions [28]. It was the first time Ukrainian military field medicine encountered such types and massiveness of injuries. While the treatment of penetrating wounds has been well described by known protocols and does not pose any particular problem, the treatment of concussions, especially their long-term effects, has its own complexities and requires special consideration.

Purpose. There is no single standardized approach to the treatment of long-term effects of concussions either in our country or anywhere else in the world. The purpose of this article is the desire of the author, based on his previous experience of treating the effects of mTBI, as well as his experience of treating combat concussions in a military field hospital with subsequent rehabilitation of such patients in civilian medical institutions, to offer, in addition to the methods already described, his own vision of how to deal with this problem using manual therapy combined with shock wave therapy.

Definition of basic concepts. This study focuses on the treatment of concussions, which are defined as a special type of general damage to the body occurring with a sudden indirect impact of, most commonly, an explosive air wave on the whole body or its extensive areas [29]. 
This type of injury occurs without damage to the external integuments of the body and appears in ICD-11 MMS (2018 version) as "intracranial trauma" (NA07) and "concussion" (NA07.0) with "loss of consciousness for a short duration of less than 30 minutes" (NA07.02) [12].

\section{Literature overview.}

Extensive literature has been studied on the definition and treatment of concussions, especially their long-term effects. An analysis of the sources allows to emphasize that although a concussion is a special type of general damage to the whole body occurring with a sudden indirect impact of, most commonly, an explosive air wave on the whole body or its extensive areas, treatment of concussions nowadays is primarily about eliminating the effects of brain injury [1-11, 13, 20, 23-25, 28].

Among the multitude of combat injuries sustained by military personnel in the course of operations in Iraq and Afghanistan, there was such a significant number of concussions that they were given a special definition to avoid any discrepancies. The American Congress of Rehabilitation Medicine (ACRM 1993) defined concussion (mTBI) as an injury with a change or loss of memory for up to 30 minutes and post-traumatic amnesia for up to 24 hours with the values of the Glasgow Coma scale being from 13 to 15 . Acute symptoms: physical - headaches, dizziness, sleep disorders; emotional - irritability, anxiety, depression; cognitive - poor concentration, memory problems [1].

Combat mTBI has been mostly covered in the English-language segment of scientific literature $[3,7,11,16,18,25,28]$. The nature of hostilities in Iraq and Afghanistan, as well as in Ukraine, contributed to an increase in the frequency of mTBI [3, 23, 28] and PTSD cases [3].

Large-scale studies by American scientists show that $15-23 \%$ of military personnel who participated in combat operations in Iraq and Afghanistan had sustained TBI, most of them being mTBI $[3,25]$. According to statistics from Walter Reed Medical Center, an estimated $60 \%$ of all blast injuries result in TBI [3]. According to Ukrainian sources, TBI accounts for 33-40\% of all combat injuries, $80 \%$ of them being mTBI, but another $30 \%$ of hidden cases have to be added here [28]. According to the data documented by UN, the number of concussions in the Ukrainian conflict as of August 2017 was over 10 thousand cases [19].

The presence of PTSD in the medical history of an mTBI victim $[11,26]$ aggravates the course of both diseases [26]. For instance, PTSD may worsen cognitive symptoms of mTBI [18]. There is a significant overlap in the neuropsychological symptoms of mTBI and PTSD, but the recovery time of these pathologies is markedly different. Most of the symptoms of mTBI disappear within a few weeks, however the symptoms of PTSD and the neuropsychological deficit associated with it may not subside over time but even increase and persist for years after the injury [3].

The long-term effects of mTBI include increased risk of developing PTSD [20], occurrence of persistent chronic pain in various areas, constant presence of which provokes a further increase in cognitive impairment [9], aggravation of sleep disorders [16]. The presence of mTBI reduces tolerance to alcohol [28], which on its own has a destructive effect on cognitive functions [17], and considering the decrease in tolerance to it, leads to a dramatic reduction in the potential for recovery [8].

The analysis of the sources studied reveals that treatment for the effects of mTBI is largely symptom oriented, but the actual foundation of such treatment is very limited and mostly focuses on "prevention through training". It involves expecting complete recovery and preventing secondary injuries; reducing associated symptoms - pain, neurological and mental disorders, sleeping problems, which may adversely affect cognitive functioning $[3,7]$.

Cognitive rehabilitation of such patients consists in the development of adaptive strategies to ensure restoration of cognitive functions [5, 13]. Compensatory and self-controlling strategies are the recommended methods for memory recovery after TBI [5, 24]. Multimodal rehabilitation offers programs that provide comprehensive treatment of cognitive and neuropsychiatric problems as well as training in interpersonal and practical skills [6]. A multidisciplinary integrative care program for veterans with symptoms of chronic mTBI, PTSD and pain, focused on training and symptom management, has been developed to maximize recovery and prevent exacerbation of symptoms [7].

To combat these pathologies, which include mTBI, PTSD and other related conditions, NICE recommended to use three techniques: selective serotonin reuptake inhibitors (SSRI), eye movement desensitization and reprocessing (EMDR) and trauma focused cognitive behavioral therapy. Among them, TF-CBT has been recognized as the most effective one [4].

At the same time, in some studies [4] the authors propose, when treating PTSD subsequent to $\mathrm{mTBI}$, to focus not on the neuropsychiatric, but on the traumatic component of the pathology and to 
use the method of vestibular rehabilitation (VR) often employed in treatment of sports injuries as a specialized method for combating vestibular disorders, characterized by dizziness, problems with balance, posture and vision [10].

Among the methods of treatment for long-term effects of mTBI proposed in the Russianlanguage segment of the sources are, most importantly, medicational methods aimed at eliminating specific symptoms. In particular, it is recommended to administer drugs of the following groups: neuroprotectors, predominantly nootropic and vascular ones [23, 27], anti-asthenic drugs, metabolic, mainly neurometabolic agents, nonsteroidal anti-inflammatory drugs, central muscle relaxants, vasoactive medications [27], antihypoxants, angioprotectors, sedatives. In addition to pharmacological agents, psychological therapy, physiotherapy, exercise therapy [23], massage and manual therapy [22] are also being suggested.

The methods for treating long-term effects of mTBI proposed so far allow to combat neuropsychiatric and traumatic symptoms, remedy to some extent emotional disturbances, perform cognitive impairment correction and rehabilitation, but they do not constitute a holistic concept of regenerating the human body and restoring it to the condition most approximate to its original state prior to the concussion.

Some sources [22] describe successful treatment of certain manifestations of the long-term effects of mTBI through manual therapy.

A concussion can over time heal without a trace, which happens relatively rarely, or cause a number of long-term medical conditions, the course of which may be much more severe than the acute phase of the concussion itself. In the medical practice of treating long-term effects of concussions, such manifestations of this condition as headaches, sleep disorders, cognitive disorders, pain in the upper and lower extremities, neuralgias, neuropathies with tunnel syndromes developing on their background and vertebral arthropathies have been observed. Chronic pain typical for long-term effects of mTBI often causes a syndrome similar to PTSD. Symptoms and diseases such as somatic pain, lumboischialgia, thoracalgia, cervicobrachialgia, cervical headaches, limited breath volume, inability to find a painless position allowing to relax, polyneuropathy-like numbness of the limbs, tunnel syndromes and epicondylitis can also be a consequence of a previous mTBI. When treating the effects of concussions, the main focus is often on brain injury and it is commonly overlooked that concussions involve damage to the whole body [29] and that, over time, for example, a whiplash neck injury sustained at the time of the concussion will lead to cervical migraine and cervicobrachialgia.

\section{Treatment of the effects of mTBI using manual therapy}

Along with the already described methods of treatment for mTBI, manual therapy is also successfully used to combat its long-term effects [22]. K. Lewit's European school of manual therapy sees the prerequisites for manual treatment in the dysfunction of the motor system. Manipulations are considered to be the most adequate method of treatment for reversible functional movement restrictions $[15$, p. 20]. The goal of manipulative treatment is to restore the function of the joint where it has been inhibited (blocked) [15, p. 23]. The objects of manipulation are functional disorders, mainly blockages in the joints. Blockage means a restriction of movement in the motor segment [15, p. 20]. "Manual therapy treats not only one specific blocked joint, but also the entire spine, being guided by the physiology and pathophysiology of the axis organ and the motor system as a whole. Regardless of whether the patient complains of cardiac, migraine-like or radicular pain, if it is considered as vertebrogenic pain, the spine should be treated in the spot and manner as required per each particular case" [15, p. 20].

Blockages are treated if they cause any complaints, as well as for prevention purposes. The techniques used to eliminate a blockage are mobilization and manipulation. Mobilization is performed by means of inhibition and facilitation of the muscles and generally involves manual techniques. It can be repeated as many times as necessary [15, p. 184]. Mobilization is performed by spring-like movements, functional or taking the form of a joint play, leading the joint to an extreme prestress position. Once such an extreme position in the blocked joint or motor segment has been reached, the mechanism of reactive muscle spasm initiates itself, interfering with the adjustment. This spasm is of a reflex nature. Physiological techniques of facilitation and inhibition and their combination can not only neutralize or reduce this interfering effect, but in certain cases even involve the muscles directly into the process of joint mobilization" [15, p. 200]. Mobilization is a preparation for manipulation. On the other hand, after 10-20 repetitions of mobilization without any application of force, it may be 
possible to eliminate the blockage even without the use of manipulation (a push). In such case, mobilization serves not only as preparation for, but also as an alternative to manipulation [15, p. 200].

"Push manipulation is a light movement of small amplitude from a fast prestress position towards the direction in which the prestress (tension) was reached or achieved by means of mobilization. This overcomes the known barrier, which naturally leads to a crack in the joint. Immediately after the push, muscle hypotension and an increase in mobility are felt" $[15, \mathrm{p} .203]$. The indications for push manipulation are as follows: incompletely eliminated blockage after mobilization, continuing reflex changes such as muscle spasms and hyperalgesia zones, including trigger points. In such cases, manipulation with minor pressure has to be applied.

Along with the restoration of joint function, manipulation can also eliminate reflex action. "Reflex muscular spasm and hyperalgesia zones disappear. Manipulations cause hypotension of muscles and connective tissue, at which point patients experience a feeling of relief and at the same time a feeling of warmth. All of this happens instantly" [15, p. 23].

In serious cases with severe restriction of movement during a chronic process, these methods are not always sufficient. In such event, a combination of separate non-specific techniques can also be used such as: traction, forced flexion, passive exercises, especially in case of radicular syndrome. Manipulations should not be frequent, but the treatment may be continued long enough if it provides good results. When comparing the simplicity and time-efficiency of manipulative treatment to the time-consumingness and high cost of other medical procedures, including medication treatment, it becomes obvious that manipulative treatment is a non-traumatic, economical and highly effective method [15, p. 184-185].

Cervical headaches, including migraines, are usually among the long-term effects of mTBI [15, p. 393-395]. They are mainly provoked by the cervicocranial syndrome which develops after concussions [15, p. 389]. But "headache is observed not only in case of a dysfunction in the upper cervical spine. Blockage in the area of the cervicothoracic transition is also a common cause of headache. The head, due to its remoteness from the center of gravity, often has to smoothen out static disturbances, the cause of which may be at the other end of the body, often in the pelvis. In such cases, it is the pelvis where treatment needs to be performed" [15, p. 390]. Therefore, it is obvious that cervical headaches as well as migraines can be triggered by both primary mTBI, which subsequently induces a cervico-cranial syndrome, causing cervical headaches, and primary spinal injuries sustained at the time of the concussion. And regardless of the localization of the areas causing cervical headaches, all of them are located within the spinal column and have to be treated by means of manual therapy.

Dizziness is usually the primary acute symptom of mTBI. Very often, it is one of the defining symptoms and long-term effects of a concussion, acquiring one or other form from mild short-term paroxysms through cervical dizziness and up to true Meniere's disease. The most common cause of various forms of dizziness, including its paroxysms in true Meniere's disease, are functional problems of the spine [15, p. 398], including those caused by the effects of a head injury or primary spinal dysfunctions sustained at the moment of a concussion. It should be noted that the cervical spine plays a significant role in patients with bouts of dizziness [15, p. 359]. Manual therapy occupies a central place in the treatment of dizziness. It provides good results in improving the condition of patients with any of its forms. If the cause is due to a vertebral factor, the condition normalizes soon after manipulative treatment has been performed [15, p. 402]. "It should be added that in most patients with Meniere's disease, there are one- or two-sided hearing impairments and decreased excitability of the labyrinth, which are absent in patients with cervical dizziness. However, neither the severity of symptoms, nor the results of treatment depend on the degree of labyrinth dysfunction. It is remarkable that dizziness is better susceptible to treatment than hearing impairment and ringing in the ears" [15, p. 402]. The problem of dizziness itself is very complicated, opinions about it are very contradictory, there are many patients suffering from dizziness, including those with long-term effects of concussions, and manual treatment is very effective for this condition [15, p. 405].

"Often, after a short acute period of injury, there is a progressive development of posttraumatic syndrome. This is very typical, for example, for concussions. In most cases of injuries to the limbs, torso and especially to the head, the accompanying damage to the spine is not being considered on time. It is always local damage that is paid attention to on the first stage and in the first place. Spinal injuries during this period can remain hidden and in most cases end up being missed. Concussion can be an example of all of the above mentioned. It is easy to imagine that when a force 
acts on the skull, damage to the cervical spine occurs at the very least. The size and mass of the skull in comparison with the cervical spine themselve indicate where the place of least resistance is, so it is not by chance that the majority of head injuries are accompanied by injuries of the cervical spine, including vertebrae fractures" $[15$, p. 423].

Cervicobrachial syndrome [15, p. 381-382], as well as epicondylitis closely related to it, is often caused, among other things, by a post-traumatic dysfunction of the cervical spine [15, p. 388]. Manual manipulations are the simplest and most effective way to treat such conditions. An injury, including mTBI, in its long-term consequences can lead to traumatic polyneuropathies, compression neuropathies, such as tunnel syndromes and radicular syndromes, all of which can be successfully treated by means of manual therapy [15].

Therefore, from the viewpoint of manual therapy, a number of diseases can be provoked by a craniocerebral and vertebral injury and are its targets for treatment [15]. Chiropractors adhere to the same opinion.

\section{Chiropractic in the treatment of mTBI.}

Chiropractic occupies a large niche in a wide field of manual medicine, primarily dealing with spinal manipulations and using methods with short levers, high speed and low amplitude. But this is only a small part of its area of interest. Outside of the spinal column, it deals with the treatment of limbs, thighs, knees, muscles, skull, nervous system and uses almost 100 methods as defined by Bergman [21]. Fundamental to chiropractic is the concept of subluxation [21] - an analogue to the concept of blockage in K. Lewit's European school of manual therapy [15, p. 23]. In today's relevant definition, formulated in 2013 by the Council on Chiropractic Practice, "subluxation is a neurological imbalance or distortion in the body associated with adverse physiological responses and/or structural changes, which may become permanent or progressive. The most frequent site for the chiropractic correction of the subluxation is via the vertebral column" [21].

Chiropractors, as well as manual therapists of K. Lewit's European school, carry out successful conservative treatment of pathologies caused by long-term effects of mTBI, especially those associated with headaches, dizziness, and disorders of the cervical spine and extremities. Numerous scientific studies by chiropractors have shown that manipulations on the spine and extremities lead to changes in cortical excitability and the resulting positive effects after manipulation on the spine are associated with downward cortical movement and cannot be explained only by changes at the spinal cord level [21]. This suggests that spinal manipulation influences the reflex arc, formed in the couse of the patient's disease.

It follows from the above that based on the great potential of the methods of manual therapy and chiropractic in treating long-term effects of mTBI, their use in combating these pathologies will allow to achieve significant results in clinical rehabilitation of patients with such disorders.

\section{Combination of manual therapy with SWT.}

As a manual therapist and traumatologist/orthopedic surgeon, the author of this article has gained more than twenty years of experience in treating the effects of traumatic brain injury using manual therapy and four years of experience in dealing with the consequences of combat concussions sustained in the course of military operations. In his medical practice, the author uses the main provisions of K. Lewit's European school of manual therapy, enhanced and supplemented by personal practice and achievements of Ukrainian colleagues. Personal experience together with the analysis of the literature studied confirms the absence of a systemic standardized approach to addressing this issue, thus giving the author reason to propose a comprehensive approach to the treatment of longterm effects of mTBI and PTSD, which does not exclude the use of traditional medications along with training and exercises. The essence of the method the author has been using for 10 years is the combination of manual therapy with shock wave therapy (SWT). As described above, manual therapy can cure or significantly alleviate the symptoms of a vast majority of pathologies caused by long-term effects of concussions and PTSD. The need to use SWT was caused by a search for more effective methods of treatment for the above pathologies. Shock wave therapy reduces pain sensitivity, improves trophic nerve function and increases blood circulation in the target area.

\section{Shock-wave therapy.}

SWT is one of the modern non-invasive means of many branches of medicine. A shock wave is a thin transition region that propagates at a supersonic speed and within which there is a sharp increase in the density, pressure and temperature of the tissue [2, 14]. The operating principle of SWT is based on the 
artificial generation of acoustic pulses - positive pressure shock waves that are not perceived by human hearing and have the following physical characteristics: short rise time - up to several nanoseconds, low frequency not exceeding $25 \mathrm{~Hz}$, pressure amplitude of 10-100 MPa as well as exponential decompression, tension and high peak pressure. The acoustic wave spreads without any delay throughout the soft tissues of the human body and gets absorbed at the border with acoustically dense tissues. Under such conditions, the power of the shock wave impact depends on the difference in tissue density.

The therapeutic effect of focused-impact shock waves is based on sequential compression and stretching of tissues with a pressure drop, which leads to the formation of cavitation bubbles, which in turn are destroyed at a maximum pressure drop and release a large amount of energy.

Although until now science cannot explain how pulsed acoustic waves influence body tissues, among the physiological impact mechanisms of shock-wave pulses studied at present, the following are known: significant increase in the intensity of local blood flow with improved vasomotor activity, enhanced angiogenesis processes with concomitant improved tissue perfusion [14], decrease in ischemia manifestations, increase in the activity of endothelial NO synthase and consequently in the synthesis of a powerful local vasodilator of nitric oxide [14], improved functioning of the lymphatic system and enhanced lymphangiogenesis.

Besides, the positive stimulating effect of low-energy acoustic pulses is also due to an increase in the synthesis and release of internal reinforcement factors (endorphins, serotonin, etc.), increased formation of growth factors, in particular, vascular endothelial growth factor [2]. Stimulation and modification of stem cells [2] and other factors also participate in the realization of enhanced regenerative processes under the influence of SWT.

Global studies have found that extracorporeal shock wave therapy (ESWT) induces neovascularization and, therefore, improves blood supply, it has been experimentally proven that the number of newly formed vessels and the expression of angiogenic markers and PCNA increase significantly after exposure to ESWT [14]. The positive effect of ESWT at the levels of intracellular VEGF has been described, and it has been proven that ESWT can increase the efficacy of cell therapy in chronic ischemia [2]. Successful use of ESWT in cardiac pathology has been shown in a number of studies.

\section{Synergistic combination of manual therapy and shock wave therapy.}

The properties of shock waves listed so far give reason to use shock wave therapy as an independent method for successful treatment of a number of pathologies associated with long-term effects of mTBI and PTSD. However, combined use of manual therapy in conjunction with shock wave therapy allows for significantly better results in terms of convalescence than the use of each of the said methods separately. Combined use of these methods enhances the effect of each of them. The novelty of the proposed method for treatment of remote effects of concussions and PTSD lies in the combination of manual therapy with shock wave therapy, where each of these two methods acts as a synergist to one another.

The method employed by the author upon collecting relevant medical history suggests:

- clarification of the localization of blocked segments and trigger points with possible use of an SWT handpiece;

- using, depending on the pathology, a radial or a focal SWT handpiece (to reduce pain sensitivity in the target area, improve trophic nerve function, enhance blood circulation) in the area of the blocked segment along the course of the discomfort causing nerve, in soft tissues, bone condyles, joints and spots where the nerve enters narrow channels. In doing so, the energy level should be maintained as tolerable as possible so that it stays comfortable for the patient;

- applying manual therapy techniques to eliminate pathologies;

- periodic repetition of these procedures to ensure a positive clinical result.

This method has been successfully applied over the past ten years and it has become particularly relevant in recent years due to the military conflict in Ukraine, which led to many thousands of concussions and PTSD cases with long-term consequences, but its effectiveness today can only be confirmed by patient reviews and their medical histories dispersed over various medical institutions the author's medical practice was associated with. Randomized controlled studies to confirm the effectiveness of the method and obtain appropriate certification have not been carried out and such studies are not even possible in an economically poor country suffering from a military aggression without external funding sources. The author sees his main task in the desire to restore health and ability to work in as many victims of war who have suffered concussions as possible and at 
the same time he is prepared to confirm the effectiveness of the method he has been practicing with no prior coverage in scientific literature by participating in any randomized controlled research project conducted anywhere in the world, addressing the treatment of long-term effects of mTBI and PTSD.

The effectiveness of the method has been determined only by subjective reviews of the victims, reduction in their complaints, elimination or reduction of symptoms, as well as the objective opinion of doctors about the clinical results of treatment, although there is also a number of instrumental methods of assessment. Most appropriate, from the author's point of view, would be the method of functional magnetic resonance imaging (fMRI).

\section{Real time functional MRI neurofeedback of as a treatment and control method}

The growth of understanding in the scientific world of the nature of functioning and correlation of brain and peripheral areas of neural circuits involved in mTBI and PTSD [15, 21, 24] allows to use realtime functional MRI neurofeedback (rt-fMRI NF) [30] both for direct treatment of the emotional and cognitive components of these pathologies and for monitoring and qualitative assessment of the treatment of their physical aspects by other methods, in particular the one proposed in this study.

It is known that $30-50 \%$ of distant mTBI and PTSD cases do not respond to pharmacological and psychological methods of treatment; for combat injuries the resistance to treatment is even higher [30]. Therefore, the development of neurobiologically sound methods of treatment and control can not only make a qualitative breakthrough in the fight against intractable pathologies, but also supplement and raise the level of medical interventions already being practiced, increasing their effectiveness and reducing resistance to treatment.

The essence of real time functional MRI neurofeedback (rt-fMRI NF) as a treatment method consists in teaching/learning to modulate the activation of target brain regions during the paradigm of symptom provocation. By training the control of emotion-related patterns of brain activity that are excited (activated) during the response of an injury, the intervention is directly aimed at the possible neural correlate of the symptoms [30].

It should be noted that this new type of intervention involving real time functional MRI neurofeedback for treatment and study of chronic PTSD was tested on a small sample of treatment-resistant war veterans and gave clinically significant results. The researchers concluded that rt-fMRI NF has the potential to facilitate the transition of neurobiological knowledge about PTSD into clinical practice [30].

When treating the effects of mTBI and PTSD using manual therapy in combination with SWT, real time functional MRI neurofeedback allows to visually observe the response of the brain to a specific targeted intervention - whether it is an impact of an SWT handpiece on the target trigger point or along the course of a specific nerve, or a mobilization/manipulation movement performed by a manual therapist. Therefore, it is possible to visually monitor and evaluate each specific intervention and the entire procedure as a whole. Moreover, this method of control, unlike anything else, will provide an opportunity to visually observe the synergistic effect of the combination of manual therapy and shock wave therapy.

Conclusions. This study analyzes the need to use a new method of treatment for long-term effects of concussions and PTSD caused by combat head injuries. This method lies in complex application of manual therapy combined with shock wave therapy. Both manual therapy and shock wave therapy can on their own solve multiple problems when dealing with the above-mentioned pathologies, but their combined power when applied within a unified method of treatment enhances the effect of each of them, creating a synergy that allows to achieve qualitatively better and significantly more successful clinical results. The novelty of this method consists in the synergistic combination of the two already recognized ways of treatment. rt-fMRI NF as a means of control allows for visual monitoring and evaluation both of each specific intervention and of the procedure as a whole, as well as visual observation of the synergistic effect of manual therapy and shock wave therapy in their combined use.

The simplicity, time-efficiency, non-invasive nature, affordability and effectiveness of this particular method when compared to the time-consumingness, high cost and limited effectiveness of other methods of treatment, including medication treatment, make its relevance pretty obvious. It should be of great interest for both health authorities and insurance companies.
Abbreviations.
mTBI - mild traumatic brain injury
PTSD - posttraumatic stress disorder
NICE - National Institute for Health and Care Excellence 
SSRI - selective serotonin reuptake inhibitors

EMDR - eye movement desensitization and reprocessing

TF-CBT - trauma focused cognitive behavioral therapy

VR - vestibular rehabilitation

ACRM - American Congress of Rehabilitation Medicine

SWT - shock wave therapy

ESWT - extracorporal shock wave therapy

PCNA - proliferating cell nuclear antigen

VEGF - vascular endothelial growth factor

CPC - circulating progenitor cells

MRI - magnetic resonance imaging

fMRI - functional magnetic resonance imaging

rt-fMRI NF - real-time-functional magnetic resonance imaging neurofeedback

$\mathrm{NF}$ - neurofeedback

\section{REFERENCES}

1. Alexander MP. Mild traumatic brain injury: pathophysiology, natural history, and clinical management. Neurology. 1995; 45:1253-1260. [PubMed]

2. Aicher A, Heeschen C, Sasaki K, Urbich C, Zeiher AM, Dimmeler S. (2006). Low-energy shock wave for enhancing recruitment of endothelial progenitor cells: a new modality to increase efficacy of cell therapy in chronic hind limb ischemia. Circulation. Dec 19; 114(25): 2823-2830.

3. Bogdanova Y, Verfaellie M, Cognitive Sequelae of Blast-Induced Traumatic Brain Injury: Recovery and Rehabilitation. Neuropsychol Rev. 2012 Mar; 22(1): 4-20.

4. Carrick FR, McLellan K, Brock JB, Randall C, Oggero E. Evaluation of the effectiveness of a novel brain and vestibular rehabilitation treatment modality in PTSD patients who have suffered combat-related traumatic brain injuries. Front Public Health. 2015; 3:15.

5. Cicerone KD, Langenbahn DM, Braden C, Malec JF, Kalmar K, Fraas M, et al. Evidence-based cognitive rehabilitation: updated review of the literature from 2003 through 2008. Archives of Physical Medicine and Rehabilitation. 2011; 92(4):519-530. [PubMed]

6. Cicerone KD, Mott T, Azulay J, Sharlow-Galella MA, Ellmo WJ, Paradise S, et al. A randomized controlled trial of holistic neuropsychologic rehabilitation after traumatic brain injury. Archives of Physical Medicine and Rehabilitation. 2008; 89(12):2239-2249. doi:10.1016/j.apmr.2008.06.017.[PubMed]

7. Defense Centers of Excellence for Psychological Health and Traumatic Brain Injury and Defense and Veterans Brain Injury Center (DCoE and DVBIC) Report of consensus conference on cognitive rehabilitation for mild traumatic brain injury; Paper presented at the Consensus Conference on Cognitive Rehabilitation for Mild Traumatic Brain Injury; Washington, DC. 27-28 April. 2009.

8. Dikmen SS, Donovan DM, Loberg T, Machamer JE, Temkin NR. Alcohol use and its effects on neuropsychological outcome in head injury. Neuropsychology. 1993; 7:296-305.

9. Dikmen SS, Machamer JE, Fann JR, Temkin NR. Rates of symptom reporting following traumatic brain injury. Journal of International Neuropsychological Society. 2010; 16(3):401411.doi:10.1017/S1355617710000196. [PubMed]

10. Herdman SJ. Vestibular rehabilitation. Curr Opin Neurol (2013) 26:96101.10.1097/WCO.0b013e32835c5ec4 [PubMed]

11. Hoge CW, McGurk D, Thomas JL, Cox AL, Engel CC, Castro CA. Mild traumatic brain injury in U.S. Soldiers returning from Iraq. The New England Journal of Medicine. 2008; 358(5):453463.doi:10.1056/NEJMoa072972. [PubMed]

12. ICD-11 for Mortality and Morbidity Statistics (ICD-11 MMS), 2018 version. https://icd.who.int/browse11/1-m/en.

13. Institute of Medicine (IOM) Cognitive rehabilitation therapy for traumatic brain injury: Evaluating the evidence. The National Academies Press; Washington, DC: 2011.

14. Ito K., Fukumoto Y., Shimokawa H., Tohoku Y. (2009). Extracorporeal shock wave therapy as a new and non-invasive angiogenic strategy. J. Exp. Med. Vol. 219: 1-9.

15. Lewit K., Sachse J., Janda V. Manual Medicine. - M.: Medicine, 1993.

16. McLay RN, Klam WP, Volkert SL. Insomnia is the most commonly reported symptom and predicts other symptoms of post-traumatic stress disorder in U.S. service members returning from military deployments. Military Medicine. 2010; 175(10):759-762. [PubMed]

17. Oscar-Berman M, Marinkovic K. Alcohol: effects on neurobehavioral functions and the brain. Neuropsychology Review. 2007; 17(3):239-257. [PMC free article] [PubMed] 
18. Polusny MA, Kehle SM, Nelson NW, Erbes CR, Arbisi PA, Thuras P. Longitudinal effects of mild traumatic brain injury and posttraumatic stress disorder comorbidity on postdeployment outcomes in National Guard soldiers deployed to Iraq. Archives of General Psychiatry. 2011; 68(1):7989.doi:10.1001/archgenpsychiatry.2010.172. [PubMed]

19. Report on the human rights situation in Ukraine 16 May to 15 August 2017. https://www.ohchr.org/Documents/Countries/UA/UAReport19th_EN.pdf, p. 7.

20. Rosenfeld JV, McFarlane AC, Bragge P, Armonda RA, Grimes JB, Ling GS. Blast-related traumatic brain injury. Lancet Neurol (2013) 12:882-93.10.1016/S1474-4422(13)70161-3 [PubMed]

21. Rosner AL. Chiropractic identity: a neurological, professional, and political assessment. J Chiropr Humanit. 2016; 23(1):35-45.

22. Shitikov, T. A. (2012). On the issue of treatment and rehabilitation of post-traumatic cephalgia using methods of manual therapy and acupressure. International Neurological Journal. 50 (4).

23. Smychek B.V., Ponomareva E.N. (2011). Traumatic brain injury: a medical and social problem. Medical news. 12.

24. Strangman GE, O'Neil-Pirozzi TM, Goldstein R, Kelkar K, Katz DI, Burke D, et al. Prediction of memory rehabilitation outcomes in traumatic brain injury by using functional magnetic resonance imaging. Archives of Physical Medicine and Rehabilitation. 2008; 89(5):974-981.doi:10.1016/j.apmr.2008.02.011. [PubMed]

25. Terrio H, Brenner LA, Ivins BJ, Cho JM, Helmick K, Schwab K, et al. Traumatic brain injury screening: preliminary findings in a US Army Brigade Combat Team. The Journal of Head Trauma Rehabilitation. 2009; 24(1):14-23. doi:10.1097/HTR.0b013e31819581d8. [PubMed]

26. Vasterling JJ, Verfaellie M. Posttraumatic stress disorder: a neurocognitive perspective. Journal of International Neuropsychological Society. 2009; 15(6):826-829. doi:10.1017/S1355617709990683.[PubMed]

27. Zakharov V.V., Drozdova E.A. Cognitive impairment in patients with traumatic brain injury. Neurology, neuropsychiatry, psychosomatics. 2013; (4): 88-93.

28. Skrypnyk O. In captivity of concussion. Mirror of the week. (2018). Issue number 12, March 31 - April 6. https://zn.ua/HEALTH/v-plenu-kontuzii-279778_.html.

29. Great medical encyclopedia / Ch. ed. B. V. Petrovsky. - 3rd ed. M.: Soviet Encyclopedia, 1974-1989. - Vol. 1-30.

30. Gerin MI, Fichtenholtz H, Roy A, Walsh CJ, Krystal JH, Southwick S and Hampson M (2016) Real-Time fMRI Neurofeedback with War Veterans with Chronic PTSD: A Feasibility Study. Front. Psychiatry 7:111. doi: 10.3389/fpsyt.2016.00111 